\title{
SMART HEALTH MONITORING AND ADVISORY SYSTEM
}

\author{
N.Dhanalakshmi ${ }^{1}$ D.Suresh ${ }^{2}$ S.Satheesbabu $^{3}$ \\ ${ }^{1}$ Professor, PSNA College of Engineering and Technology, Dindigul, India \\ 2 Professor, PSNA College of Engineering and Technology, Dindigul, India \\ ${ }^{3}$ Associate Professor, PSNA College of Engineering and Technology, Dindigul, India \\ ndmugi@gmail.com
}

\begin{abstract}
Health has quintessential significance in our day to day life. Health is the degree of beneficial and metabolic efficiency of dwelling organisms..The motive of this task is to boost fitness alert machine for elders and bodily challenged people. The objective is to hint patient's fitness with the help of sensors."The Health Alert Scheme" can preserve track of patient's pulse rate, eco heart rate of, pressure stage rate, temperature via the usage of the respective sensors. The Sensors are interfaced with the controller Arduino UNO board. The Arduino microcontroller which is used for processing the data. The computerized messages to preserve their food plan is additionally send to their caretakers. Once fitness problem is detected, call signals are send to the caretakers. This is the best way to display the sufferers for the medical doctors from any area at anytime.
\end{abstract}

Keywords: Sensors, Arduino UNO, Health alert System, Monitoring.

\section{INTRODUCTION}

Monitoring of Patients has turn out to be a difficult for the previous few years. Because of this problems humans are uncovered to hazardous issues. Not solely in hospitals however additionally the non-public fitness caring amenities are opened by using the IoT technology. Early detection of persistent illnesses can be handy with these technology. The physique temperature, heart rate, blood pressure, eco price of coronary heart are high parameters to diagnose the disease. So the thinking is to boost a fitness monitoring system with the aid of the use of IOT. It can also will increase get admission to to fitness offerings and reducing cost. Whenever there are fluctuations from the regular value, it sends food regimen data to the caretakers. The affected person checking monitoring is one of the substantial enhancements as a end result of its innovative innovation.

\section{II.MOTIVATION}

In rural hospitals, the amenities for fitness caring are limited. The bad high-quality of fitness administration allows troubles in fitness care machine Everyone need to get the expertise of very own fitness as handy and early as possible. Also it need to be really worth for every .Latest file of The India Spend evaluation of records says that the 500,000 medical practitioner scarcity in India. WHO defines the physician affected person ratio will be 1:1000 which has been failed in India.In growing nations there is lack of sources and administration to attain out the issues of individuals. A frequent man can't come up with the money for the luxurious and day by day test up for his health. For this motive more than a few structures which supply handy and certain caring unit has been developed. Theses machine reduces time with safely dealt with equipment. 


\section{III.RELATEDWORK}

Smart Elderly Home Monitoring Scheme with an Android Phone (2013) by way of Kenny T.H.Chieng et al., that considers or takes into account sure facts, which are coronary heart assault and stroke as they are the main purpose of hospitalization of the elder people. There is greater probabilities of survival if the older humans receives the remedy inside an hour. it had additionally been developed. An android clever cellphone with accelerometer is used to observe a fall of the carrier, and this android system is recognised as healthcare device.

The android cellphone is then related to the monitoring machine with the aid of the use of the TCP/IP protocol via Wi-Fi. Because of this system, aged and chronically unwell sufferers can continue to be independently in their very own domestic and invulnerable in the understanding that they are being monitored.The downside of this gadget is that it solely considers elder human beings as there is extra possibilities of unexpected (emergency situation) outbreak in them like coronary heart assault and stroke. Monitoring and tracking of IoT healthcare Akhtar Rasool proposed continuous real time monitoring in a survey conducted by Subhra Shriti Mishra. The use of RFID tags can be completed in clinical devices spherical to monitor the clock. Especially when the place packaging of medicinal products is automated, readers installed in the manufacturing line will spontaneously recognize and convert the statistics on all medicines. These readers are able to save all relevant statistics on the methods and can monitor everything in circulation at any time. This can also be completed for the storage environment.

The disadvantage of the device is that it solely concentrates on elder human beings and it consists of shirt for sporting which value a lot. Survey of Health Monitoring Management Using IoT (2016) via S. et al., : The Smart Healthcare machine collects the physiological parameter values such as ECG, Temperature and Heart charge of the patient. Based on the parameter values, the selection guide device will figure out the emergency situation. The clever healthcare device will grant Mobile app notification about the emergency state of affairs of the affected person to the doctors. The disadvantage of the gadget is that the notification will be ship solely if there is a web connection. Arduino UNO and GSM based totally wi-fi fitness monitoring gadget for sufferers by means of Pratiksha W.Digarse;SanjayKuamar L.Patil proposed a clever embedded machine machine which video display units sufferers fitness continuously. This gadget video display units sufferers coronary heart rate, physique temperature. If any of the above parameters goes past the threshold value, this clever system informs medical doctors or care taker and ask for corrective moves to retailer sufferers life.

\section{IV.Proposed System}

$>$ The objective of this paper is implementing the Health monitoring system.

$>$ The SMS and CALL alert will send to the predefined persons 


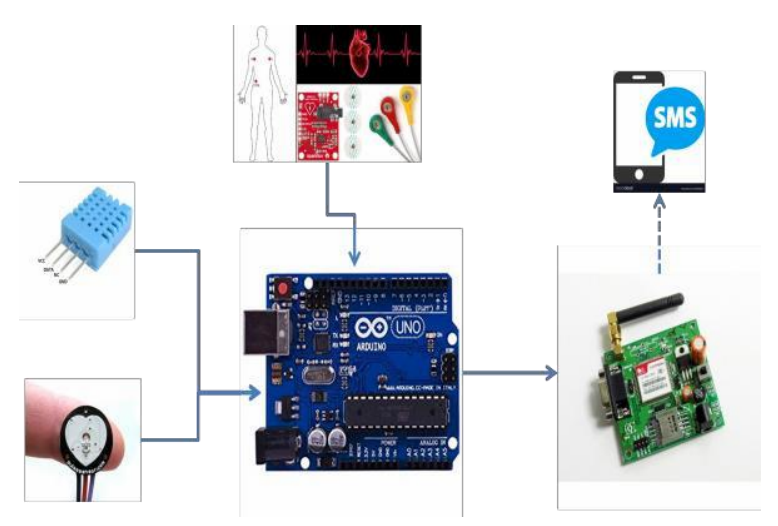

Figure 1: Proposed model

In the above sections, the components of the intelligent boards system are used to monitor the patient's body parameters. Raw data from the health parameters of patients will be sensed and transmitted using GSM technology to the back-end server application.

\section{MODULES:}

- Temperature Sensor

- Pulse Sensor

- ECG

- GSM

- Arduino

\section{Working Flow of the System}

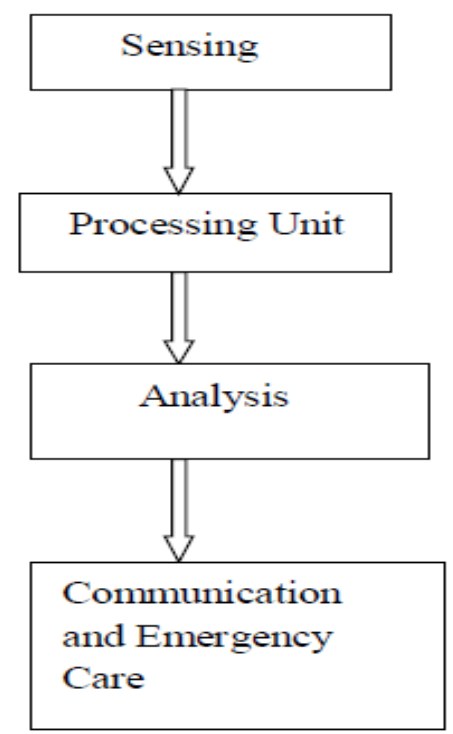

Figure 2: Flow digram of the Working System 


\section{TEMPERATURESENSOR}

The DHT 11 is used for a temperature sensor which requires a thermocouple or an RTD to measure the temperature by an electric sign (Resistance Temperature Detectors).

\section{PULSE SENSOR:}

The new model uses the optical photoplethysmography reflective sensor TCRT1000. The use of TCRT100 simplifies the sensor phase construction system, as each mild infra round emitter diode and the detector is organized as facets in a led package, thereby blocking the surrounding ambience light, which should affect the sensor performance in all other cases. The output is a digital pulse synchronized with the heartbeat. The output pulse can be supplied to an ADC channel or a microcontroller digital pin to further process and retrieve the heart price in beats per minute (BPM).

\section{ELECTROCARDIOGRAM}

A test that measures the electric heartbeat effort is an electrocardiogram - abbreviated as an EKG or an ECG. An electric pulse (or "wave") travels through the heart with every beat[4]. This wave causes the muscle to pump blood from the heart and to squeeze. Two main types of information are presented in an ECG. The health practitioner can decide how long electrical waves take to circumvent the heart by measuring time intervals on the ECG. Electrocardiography is the means of the electrification of the coronary heart by the use of electrodes positioned on your skin, a recording - a sketch of voltage versus time[5]. Only the ECG is informed by the computer. The energy is not shipped into the body. It doesn't harm us, therefore.

\section{Working of Electrocardiography}

Electrocardiographs are recorded by means of machines which are connected to a central unit by electrodefinition. Electrocardiograms convert the electrical coronary heart to a digital signal using analog-to-digital converters. ECG registration is a safe and painless operation [18]. The machines are powered by hands, but have several safety points including a grounded lead. they are designed with a lot of strength. Electronic interpretation algorithms are the most modern ECG machines. Facetten such as PR interval, QT, corrected QT interval (QTc), axis PR, QRS axis, rhythm and more are calculated.

\section{GSM MOSDULE:}

For mobile communication, GSM stands for a Global System. Today, GSM, which represents 70 percent of todayâ s Digital Wi-Fi market, is employed by more than 800,000 customers across 10090 nations. In GSM, the geographical location is divided into hexagonal cells with a facet based on transmitter strength and transmitter charge (number of cease user). There is a base station in the heart of the cell consisting of a transceiver (combination of an antenna and a transmitter). GSM is a multiple access, multiple access, frequency access and hopping aggregate of TDMA (time division). GSM is a multiple access.

\section{WORKING:}

GSM is a blend of Multiple Access Time (TDMA) and Multiple Access (FDMA) and Hopping Frequency. GSM initially uses two $25 \mathrm{MHz}$ frequency bands: 890 to $915 \mathrm{MHz}$ up-link and 935 to 960 MHz down-link frequency. Two $75 \mathrm{MHz}$ band were added later. Later. The up-link from 1710 to 1785 $\mathrm{MHz}$ and down-links from 1805 to $1880 \mathrm{MHz}$. Up-link is the hyperlink from the basement to the 
satellite TV for the PC, and down-link from a satellite TV for the PC to one or more floor or receiver stations. It splits the $25 \mathrm{MHz}$ band into 124 channels, each of which has a width of $200 \mathrm{KHz}$, and closes $200 \mathrm{KHz}$ as a defense band to prevent interference.

\section{MICROCONTROLLER:}

ATMega Microcontroller Introduction ATMega microcontrollers are owned and manufactured by Atmel Corporation in the AVR microcontroller family. An 8-bit ATMega Microcontroller is based on primarily Harvard Architecture, with Reduced Instruction Set (RISC).It features trendy points such as the on-chip ROM (read memory only), data RAM (river access emory), EEPROM facts, input/output ports and more peripherals (electrolytic removable read only), analog to digital converters (ADC), serial interface ports, etc. The chip has fashionable points. They have a hundred 20th and a higher level of practice and remind you of $4 \mathrm{~K}$ to $256 \mathrm{~K}$ bytes.

ATMega microcontrollers are entirely based on separate memory and application information. Flash Random Access Memory is also an important reminiscence of program or code memory (ROM). Software reminders of $8 \mathrm{~K}$ to $128 \mathrm{~K}$ octets measurement. The statistic memory is split into three components - 32 registers of general purposes, input/output memory and internally static random access memory (SRAM). The I/O Memory and Internal SRAM measures vary from chip to chip, while the dimension of the General Purpose registers is fixed [9-12].

\section{APPLICATIONS:}

- Fall and Movement detection applications

- Medication consumption monitoring applications

- Location monitoring applications

- Day-to-Day activity monitoring applications

\section{V.CONCLUSION:}

The wireless health monitoring machinery from Arduino UNO and GSM is the most appropriate science, because it sends SMS fast and conveniently and can also work over long distances at very small costs, to evaluate current technological know-how. The medical practitioner can easily act if any essential conditions occur within biomedical parameters of the patient, and heart beat (meter beat), physical temperature and the saline degree are measured. If the one parameter is affected, it will also send the clinical recommendations to the caretaker. When the person concerned is integral, the CALL will ship.

\section{REFERENCES:}

[1]. Srinivasan Subramanian, K.Vinoth Kumar, T.Jayasankar, Malladi Srinivas, "Automated Kitchen Management and Provisions Monitoring System Using IoT Technology", International Journal of Control and Automation, Vol.13, No.2, (2020), pp.776-784.

[2]. MikhailSt-Denis,LifeLine,Accessed:(29/11/2015), availablehttp://wwwmik hailstdenis.com /projects/ personalLifeLine.html.

[3]. Eckerson, Wayne W. "Three tier client/server architectures: achieving scalability, performance, and efficiency in client/server applications."Open Information Systems 3.20 (1995): 46-50.

[4]. A. Sheryl Oliver, Kavithaa Ganesan, S. A. Yuvaraj, T. Jayasankar, Mohamed Yacin Sikkandar \&N. B. Prakash, Accurate prediction of heart disease based on bio system using regressive 1114 
learning based neural network classifier, Journal of Ambient Intelligence and Humanized Computing (2020), https://doi.org/10.1007/s12652-020-02786-2

[5]. Stephe.S, T.Jayasankar, "Motor Imagery Recognition of EEG Signal using Cuckoo-Search Masking Empirical Mode Decomposition", International Journal of Innovative Technology and Exploring Engineering (IJITEE), Vol.8, Issue-11, September 2019, pp.2717-2720.

DOI: 10.35940/ijitee.K2175.0981119

[6]. Behance,LifeLine on Behance, Accessed: (13/11/2015), available a http://www.behance.net/Gallery/LifeLinea/321 119.

[7]. Angel,Lumo Run - Revolutionary Smart Running Shorts, Accessed:(13/11/2015), available at http://www.lumobodytech.com/.

[8]. Thepu,how mobile is making us healthier, Accessed: (13/11/2015), available at http://thepu.sh/trends/take-two-wearables-and- call-me-in-the-morning-how-mobile-is-making- ushealthier/.

[9]. Arduino Uno,Arduino - ArduinoBoardUno, Accessed: (13/11/2015), available at https://www.arduino.cc/en/Main/ArduinoBoard Uno.

[10]. 4project,Pulsesensor, Accessed:(13/11/2015),availablehttps://www.4project.co.il /product/pulsesensor

[11]. Dash.co.il,LilyPad Temperature Sensor, Accessed: (13/11/2015), avail-able http://goo.gl/xnglsv

[12]. S.Krishnaveni,S.Senthilraja,T.Jayasankar,P.SathisBabu, "Analysis and control of the motor vibration using arduno and machine learning model," Materials Today Proceedings (Elsevier 2020), https://doi.org/10.1016/j.matpr.2020.11.261 